\title{
On the inclusion of a velocity-dependent basal drag in avalanche models
}

\author{
J. M. N.T. GRAY, Y. C. TAI \\ Institut für Mechanik, Technische Universität Darmstadt, D-64289 Darmstadt, Germany
}

\begin{abstract}
The Savage-Hutter model is generalized by including a velocity-dependent drag in addition to the usual Coulomb dry friction at the base of the avalanche. Both linear and quadratic velocity dependencies are considered, with either constant or asymptotically constant drag coefficients for large thickness $h$. The singular nature of the constant coefficient model for small $h$ is demonstrated and it is shown that the asymptotic model allows the tail of the avalanche to move at a finite velocity. The inclusion of velocity drag changes the stress state in the avalanche and new earth-pressure relations are derived and investigated.
\end{abstract}

\section{INTRODUCTION}

In the Savage and Hutter $(1989,1991)$ model for the flow of dense granular materials, such as snow, ice or rock avalanches, the Coulomb dry friction at the base does not increase in response to increased flow rates. As a result, the velocity of an accelerating avalanche does not tend to a finite limit but continues to increase without bound. This is physically unrealistic. Velocity-dependent basal-drag laws have therefore been used in both point mass (e.g. Voellmy, 1955) and continuum models (e.g. Hutter and Greve, 1993) to bring the avalanche to a steady terminal velocity. In this paper some of the features of these models are discussed.

\section{GOVERNING EQUATIONS}

In the Savage-Hutter (1991) theory, curvilinear coordinates $(x, z)$ were defined, which lie parallel to, and normal to, the local slope topography. The leading-order depth-integrated mass balance is

$$
\frac{\mathrm{d} h}{\mathrm{~d} t}+h \frac{\partial u}{\partial x}=0
$$

where $h$ is the avalanche thickness, $u$ is the down-slope velocity and $\mathrm{d} / \mathrm{d} t=\partial / \partial t+u \partial / \partial x$ is the total derivative. The leading-order depth-integrated momentum balance is

$$
\rho h \frac{\mathrm{d} u}{\mathrm{~d} t}=\rho g h \sin \zeta+\tau-\rho g h \cos \zeta K_{x} \frac{\partial h}{\partial x}
$$

where $\rho$ is the avalanche density, $g$ is the gravitational acceleration, $\zeta$ is the local slope inclination angle, $\tau$ is the basal shear traction and $K_{x}$ is the earth-pressure coefficient.

The basic idea behind the generalization of the SavageHutter model is to decompose the basal shear stress $\tau$ into two contributions:

$$
\tau=\tau_{c}+\tau_{u} .
$$

The first contribution $\tau_{c}$ is simply the conventional Coulomb dry-friction law and the second term $\tau_{\mathrm{u}}$ is an additional velocity-dependent drag relation, that is

$$
\left.\begin{array}{l}
\tau_{\mathrm{c}}=-(u /|u|) p_{z z}^{b} \tan \delta, \\
\tau_{\mathrm{u}}=-\rho\left(c_{1} u+c_{2}|u| u\right)
\end{array}\right\}
$$

where $p_{z z}^{\mathrm{b}}$ is the normal pressure at the base of the avalanche, $\delta$ is the basal angle of friction and $c_{1} \geq 0$ and $c_{2} \geq 0$ are linear and quadratic drag coefficients, respectively. Savage and Hutter (1991) showed that to leading order the normal basal pressure $p_{z z}^{\mathrm{b}}=\rho h\left(g \cos \zeta+\kappa u^{2}\right)$, where $\kappa$ is the local curvature of the chute. In this paper it is assumed that $\kappa=0$ for simplicity.

Two models are considered for the linear and quadratic drag coefficients $c_{1}$ and $c_{2}$. The first is the simplest possible model in which the drag coefficients are equal to the constants $c_{1}^{*} \geq 0$ and $c_{2}^{*} \geq 0$, respectively. The second model assumes that for larger avalanche thicknesses the drag coefficients approach the same constant values but that for smaller avalanche thicknesses $c_{1}$ and $c_{2}$ are proportional to $h$. That is,

$$
\begin{aligned}
& \text { (1). } \quad c_{1}=c_{1}^{*} \text {, } \\
& \text { (2). } \left.\quad c_{1}=c_{1}^{*} h /\left(h_{1}+h\right), \quad \begin{array}{l}
c_{2}=c_{2}^{*}, \\
c_{2}^{*} h /\left(h_{1}+h\right)
\end{array}\right\}
\end{aligned}
$$

where the parameter $h_{1}$ is constant. Note, that the first of these models is a special case of the second, as the latter reduces to the former when $h_{1}=0$. For ease of reference, the first model, and the second model with $h_{1}=0$, is termed the constant coefficient model and the second model with $h_{1}>0$ is called the asymptotically constant model or asymptotic model.

On substituting the drag relations (4) into Equation (2), the depth-integrated momentum balance reduces to

$$
h \frac{\mathrm{d} u}{\mathrm{~d} t}=g^{\prime} h-\left(c_{1} u+c_{2}|u| u\right)-g h \cos \zeta K_{x} \frac{\partial h}{\partial x}
$$

where the driving force

$$
g^{\prime}=g \cos \zeta(\tan \zeta-(u /|u|) \tan \delta)
$$

For small avalanche thicknesses the constant and asymptotic models have a very different behaviour. Provided the solutions remain regular, the limit as $h \rightarrow 0$ of the momentum balance in Equation (6) is

$$
\left.\begin{array}{rlrl}
c_{1}^{*} u+c_{2}^{*}|u| u & =0, & & h_{1}=0, \\
0 & =0, & & h_{1}>0,
\end{array}\right\}
$$

for the constant coefficient and asymptotic models, respectively. It follows that for the constant coefficient model the velocity $u=0$ at all points where the avalanche thickness $h=0$, i.e. at the boundaries of a finite-mass avalanche. These boundaries can only move if the derivatives $\partial h / \partial x$ 
and/or $\partial u / \partial x$ become singular. Whereas in the asymptotic model, and the original Savage-Hutter theory, the momentum balance is trivially satisfied at $h=0$ and the velocity is determined by continuity.

Typical velocity magnitudes within the interior of a finite-mass avalanche can be estimated from the case of steady uniform flow, in which $\partial / \partial t=0$ and $\partial / \partial x=0$. Assuming that the avalanche is being accelerated downslope, $\delta \leq|\zeta| \leq \pi / 2$, and that the velocity has the same sign as the slope inclination angle, $\operatorname{sgn}(u)=\operatorname{sgn}(\zeta)$, then Equation (6) yields the quadratic equation

$$
c_{2} u_{\mathrm{s}}^{2}+c_{1} u_{\mathrm{s}}-\left|g^{\prime}\right| h=0,
$$

for $u_{\mathrm{s}}$, the modulus of the steady uniform flow velocity. Substituting the asymptotic drag coefficients from Equations (5), it follows that

$$
\left.\begin{array}{ll}
u_{\mathrm{s}}=\frac{-c_{1}^{*}+\sqrt{\left(c_{1}^{*}\right)^{2}+4\left|g^{\prime}\right| c_{2}^{*}\left(h_{1}+h\right)}}{2 c_{2}^{*}}, & c_{2}^{*} \neq 0, \\
u_{\mathrm{s}}=\left|g^{\prime}\right|\left(h_{1}+h\right) / c_{1}^{*}, & c_{2}^{*}=0 .
\end{array}\right\}
$$

The modulus of the steady uniform velocity $u_{\mathrm{s}}$ is plotted as a function of the avalanche thickness $h$ in Figure 1 for each of the models. For large avalanche thicknesses, the quadratic drag law implies that the steady velocity $u_{\mathrm{s}} \sim \sqrt{h}$, whilst with the linear drag law $u_{\mathrm{s}} \sim h$, for both constant and asymptotic models. It follows that, in the absence of other effects, thicker parts of the avalanche will tend to move faster than thinner sections of the avalanche and therefore non-linear waves (e.g. Whitham, 1974) and shocks are expected. In the limit as $h \rightarrow 0$, the steady uniform velocity $u_{\mathrm{s}} \rightarrow 0$ for the constant coefficient model, whilst in the asymptotic model it tends to a constant finite limit $u_{\mathrm{s}}^{\min }=\left.u_{\mathrm{s}}\right|_{h=0}$. This implies that with the asymptotic model the rear of the avalanche (where an expansion wave devel-
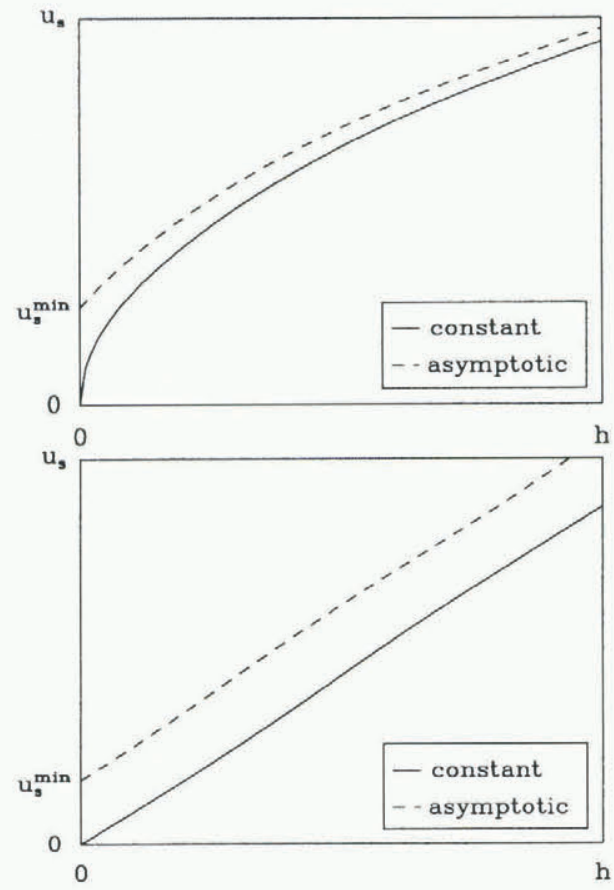

Fig. 1. The modulus of the steady uniform velocity $u_{\mathrm{s}}$ is plotted as a function of $h$ for the quadratic (top) and linear (bottom) drag laws, and for both the constant coefficient (solid) and asymptotic (dashed) models. ops) moves with a finite velocity, whilst it remains fixed at its initial position with the constant coefficient model (Fig. 2).

\section{EARTH-PRESSURE COEFFICIENTS}

The inclusion of a velocity-dependent drag increases the applied shear traction at the base of the avalanche, and will therefore alter the stress state within the avalanche. This effect has not been considered in other generalizations of the Savage-Hutter theory. In this paper, the simple arguments of Savage and Hutter (1989) are paralleled to derive a new earth-pressure coefficient $K_{x}$ appropriate for velocity-dependent drags.

It is assumed that the same simple stress state prevails in the granular material. That is, one of the principal stresses, $p_{y}$, lies perpendicular to the plane of avalanche motion and the magnitude of one of the remaining principal stresses, $p_{x}$ or $p_{z}$, is equal to $p_{y}$. Thus, the three Mohr-stress circles collapse to a single Mohr circle, with principal stresses, $p_{x}, p_{z}$, in the $x z$-plane. This stress state can be conveniently visualized on a Mohr circle diagram. All the allowable stress states lie on the circle

$$
(p-a)^{2}+\tau^{2}=r^{2}
$$

with radius $r$ and centre $a=\left(p_{x x}+p_{z z}\right) / 2$. The principal stresses, $p_{x}$ and $p_{z}$, lie on the $p$ axis as illustrated in Figure 3, and stress state $\left(p_{z z}, \tau_{x z}\right)$ lies diametrically opposite $\left(p_{x x}, \tau_{x z}\right)$.

The snow is assumed to be a Mohr-Coulomb material that satisfies the yield criterion

$$
\tau \leq \pm p \tan \phi
$$

where $\phi$ is the internal angle of friction. This corresponds to the domain between the two straight lines on the Mohr-circle diagram inclined at $\pm \phi$ to the $p$ axis and intersecting at the origin. When the material is at yield the Mohr circle of stress is tangent

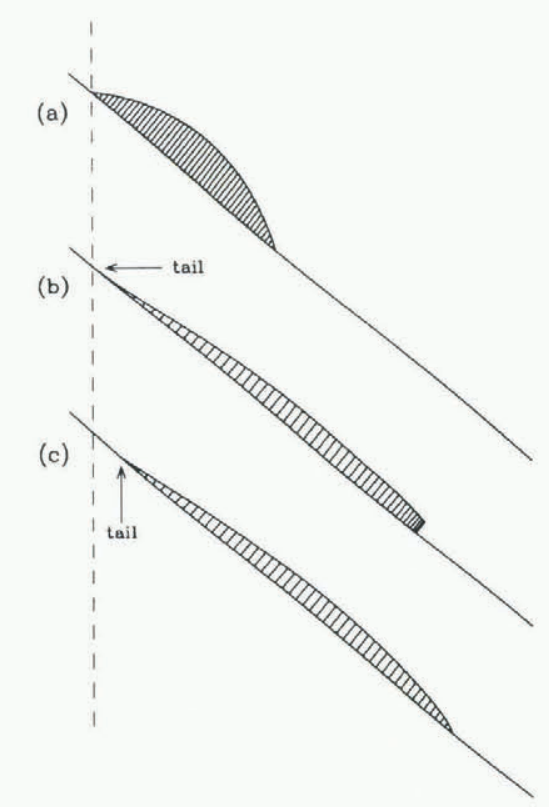

Fig. 2. A finite mass of granular material is released from rest on an inclined plane (a) and flows downslope. With the constant coefficient model ( $b$ ) the tail of the avalanche remains fixed at its initial position and singular gradients occur at the front. With the asymptotic model (c) the tail moves with a finite velocity and the gradients at the front remain regular. 


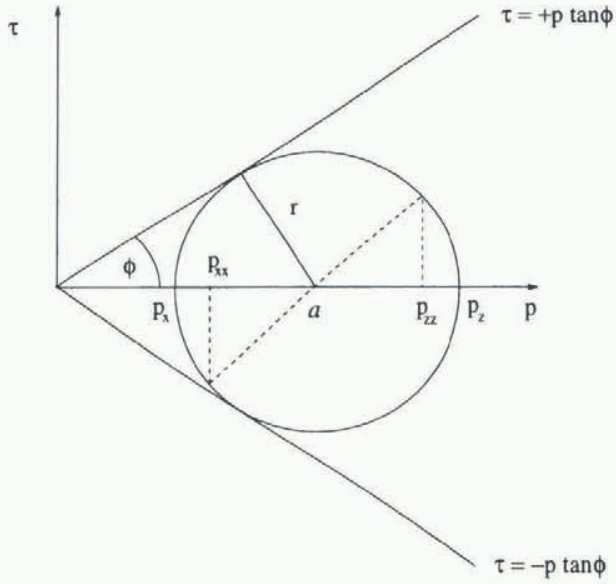

Fig. 3. The stress state within the avalanche is represented on a Mohr-circle diagram. The yield criterion corresponds to the two straight lines inclined at angles $\pm \phi$ to the horizontal. When the material is at yield the Mohr stress circle is tangent to the yield lines.

to the Mohr-Coulomb lines $\tau= \pm p \tan \phi$, as illustrated in Figure 3, and by elementary trigonometry it follows that

$$
r=a \sin \phi .
$$

The position of the centre of a Mohr circle which satisfies the yield criterion (12) is obtained by substituting Equation (13) into Equation (11) and solving the quadratic to give

$$
a=\sec ^{2} \phi\left(p \pm \sqrt{p^{2} \sin ^{2} \phi-\tau^{2} \cos ^{2} \phi}\right) .
$$

The earth-pressure coefficient $K_{x}$ relates the limiting normal stress in the $x$ and $z$ directions, and was defined by Savage and Hutter (1989) as

$$
K_{x}=p_{x x} / p_{z z} .
$$

The in-plane pressure $p_{x x}$ can be eliminated by recalling that by definition, $a=\left(p_{x x}+p_{z z}\right) / 2$, and that at yield $a$ is given by Equation (14). It follows that the earth-pressure coefficient for Mohr-Coulomb material at yield is

$$
K_{x}=2 \sec ^{2} \phi\left\{1 \pm \sqrt{\sin ^{2} \phi-\frac{\tau_{x z}^{2}}{p_{z z}^{2}} \cos ^{2} \phi}\right\}-1 .
$$

Savage and Hutter (1989) used the leading-order basalstress state to determine the earth-pressure coefficient, $K_{x}^{\mathrm{b}}$, at the base of the avalanche. To leading order the basal shear stress $\tau_{x z}^{\mathrm{b}}=\tau_{\mathrm{c}}$ and the normal pressure $p_{z z}^{\mathrm{b}}=\rho g h \cos \zeta$ in their model. In this paper, the Coulomb dry friction $\tau_{\mathrm{c}}$ is supplemented by an additional velocity dependent drag $\tau_{\mathrm{u}}$, so that to leading order the basal shear stress $\tau_{x z}^{\mathrm{b}}=\tau_{\mathrm{c}}+\tau_{\mathrm{u}}$, whilst the normal basal pressure remains the same as above. The earth-pressure coefficient at the base of the avalanche is therefore modified. Savage and Hutter (1989) also assumed that the earth pressure coefficient remained approximately constant through the depth of the avalanche, i.e. $K_{x}=K_{x}^{\mathrm{b}}$, which is also assumed here. It follows that for a Mohr-Coulomb material subjected to both Coulomb dry-friction and velocity-dependent drag laws the earth-pressure coefficient is

$$
K_{x_{\text {act } / \text { pas }}}=2 \sec ^{2} \phi\{1 \mp \sqrt{\Psi}\}-1
$$

where

$$
\Psi=\sin ^{2} \phi-\left(\tan \delta+\frac{c_{1}^{*}|u|+c_{2}^{*}|u|^{2}}{g\left(h+h_{1}\right) \cos |\zeta|}\right)^{2} \cos ^{2} \phi
$$

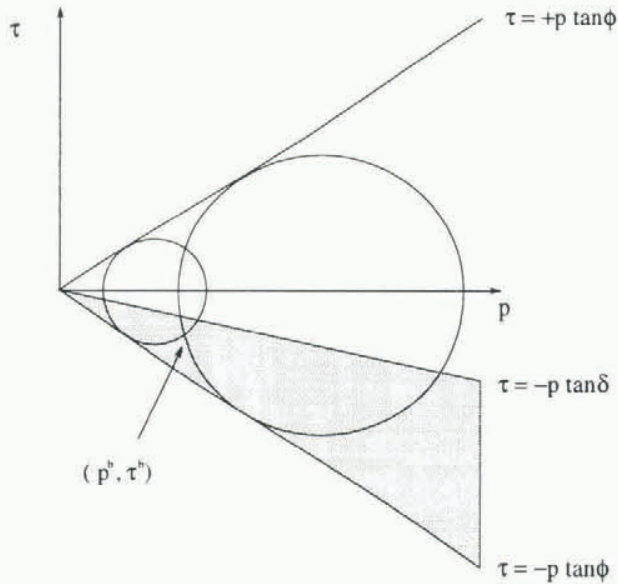

Fig. 4. The basal stress $\left(p^{\mathrm{b}}, \tau^{\mathrm{b}}\right)$ lies in the shaded region of the Mohr-circle diagram, instead of on the Coulomb dry-friction line $\tau= \pm p \tan \delta$ in the Savage-Hutter model. The two Mohr circles through this point correspond to the passive and active stress states.

is a function of avalanche thickness and velocity. This is an important new feature of the model. The change in the assumed stress state is illustrated schematically in Figure 4. The basal stress $\left(p^{b}, \tau^{b}\right)$ no longer lies on the Coulomb dryfriction line $\tau= \pm p \tan \delta$, but, because of the additional velocity-dependent drag, it is defined within the whole of the shaded region.

The earth-pressure coefficient can take two limiting values, $K_{x_{\text {act }}}$ and $K_{x_{\text {pas }}}$, corresponding to whether the stress state is active or passive. Savage and Hutter (1989) introduced the ad hoc definition that a dilatation occurs in the active regime and a compression in the passive regime, i.e.

$$
K_{x}= \begin{cases}K_{x_{\text {uct }}} & \partial u / \partial x \geq 0 \\ K_{x_{\text {pas }}} & \partial u / \partial x<0,\end{cases}
$$

although other definitions are possible (Tai and Gray, 1998). The larger of the two circles in Figure 4 corresponds to the passive stress state and the smaller to the active stress state.

In order to obtain real values of the earth-pressure coefficient, the function $\Psi$ in Equation (18) must be greater or equal to zero. In the original Savage-Hutter theory, when $c_{1}^{*}=c_{2}^{*}=0$, the function $\Psi \geq 0$ provided $\delta \leq \phi$ and the active and passive stress states are defined for all values of $h$ and $u$

$$
K_{a, p}=2 \sec ^{2} \phi\left(1 \mp\left\{1-\cos ^{2} \phi \sec ^{2} \delta\right\}^{\frac{1}{2}}\right)-1 .
$$

Here, the conditions are considerably stricter. For fixed values of the velocity modulus, $|u|$, the function $\Psi$ is positive if and only if

$$
\left.\begin{array}{lll}
\text { (1). } & h \geq h_{0}, & h_{1}=0, \\
\text { (2). } & h \geq h_{0}-h_{1}, & h_{1}>0,
\end{array}\right\}
$$

where

$$
h_{0}=\frac{c_{1}^{*}|u|+c_{2}^{*}|u|^{2}}{g \cos |\zeta|(\tan \phi-\tan \delta)} \geq 0 .
$$

Thus, for the constant coefficient model the avalanche thickness must be greater than some positive thickness $h_{0}$ throughout the whole of the avalanche domain in order to obtain a well-posed problem. For a finite-mass avalanche, where there are necessarily regions close to the boundaries 

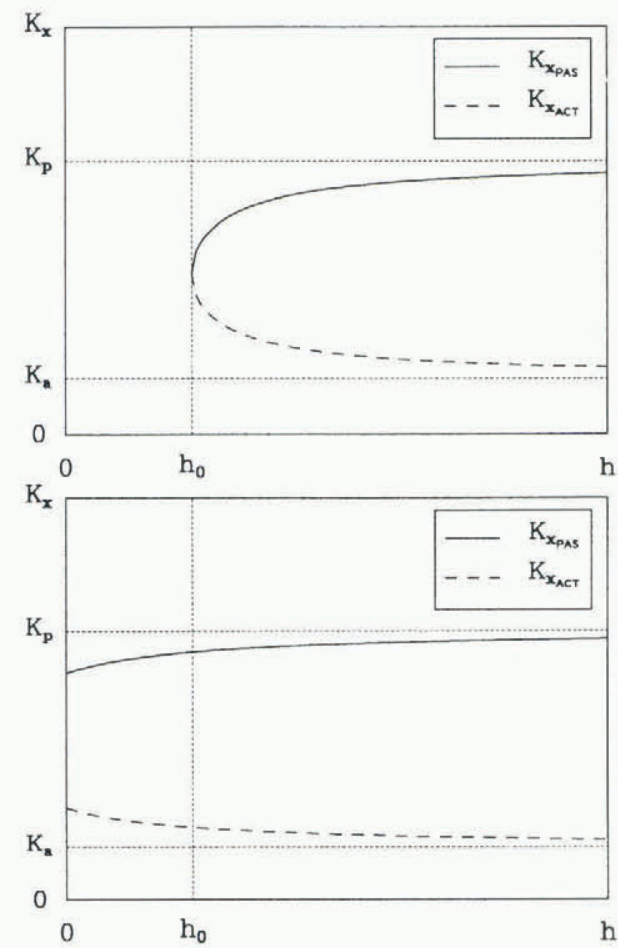

Fig. 5. The active (solid) and passive (dashed) earth-pressure coefficients are plotted as a function of $h$. The constant coefficient model (top) contains a region for small $h$ where $K_{x}$ is not defined. When $h_{1} \geq h_{0}$ the earth pressure is defined for all $h$ with the asymptotic model (bottom).

where $h<h_{0}$, the earth-pressure coefficient is complex and the problem is ill-posed. For a finite-mass avalanche, in which $h \geq 0$, the asymptotic model is well-posed if $h_{1} \geq h_{0}$ and is ill-posed if $h_{1}<h_{0}$. The domains in which the earth pressure is defined are illustrated in Figure 5.

For fixed values of the avalanche thickness $h$ the function $\Psi$ is greater or equal to zero provided

$$
|u| \leq u_{m}
$$

where the maximum velocity

$$
\left.\begin{array}{ll}
u_{\mathrm{m}}=\frac{-c_{1}^{*}+\sqrt{\left(c_{1}^{*}\right)^{2}+4|\hat{g}| c_{2}^{*}\left(h_{1}+h\right)}}{2 c_{2}^{*}}, & c_{2}^{*} \neq 0, \\
u_{\mathrm{m}}=|\hat{g}|\left(h_{1}+h\right) / c_{1}^{*}, & c_{2}^{*}=0
\end{array}\right\}
$$

and

$$
\hat{g}=g \cos |\zeta|(\tan \phi-\tan \delta) .
$$

That is, for both the constant coefficient and asymptotic models, with either linear or quadratic drag laws, there is an upper limit to the allowable velocity magnitude. Above this limit the earth pressure is not defined and the model is ill-posed. This is illustrated in Figure 6 for the case of quadratic drag. Indeed, if $\phi=\delta$ then the shear stress will exceed that allowed by the Mohr-Coulomb criterion as soon as the velocity becomes non-zero.

\section{GONCLUSIONS}

The asymptotic model for the velocity-dependent drag coefficients has a number of points to recommend it above the constant coefficient model. First, the singular nature of the solution at the front of the avalanche is removed and, secondly, the tail of the avalanche can move with a finite org/10.3189/1998AoG26-1-277-280 Published online by Cambridge University Press

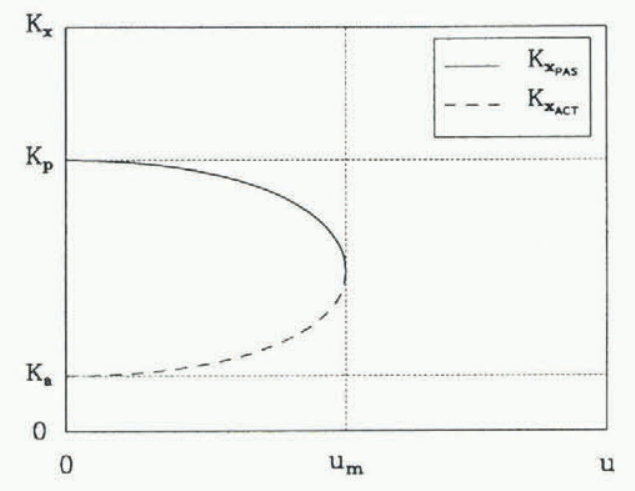

Fig. 6. The active (solid) and passive (dashed) earth-pressure coefficients are plotted as a function of $u$ for the quadratic drag law.

velocity. Not only is this good for numerical methods but it is also physically more realistic. In addition, if the new earth-pressure coefficient (Equation (17)) is used, then the asymptotic model ensures that $K_{x}$ is well defined for small avalanche thicknesses, provided that the parameter $h_{1}$ is suitably chosen.

The velocity-magnitude restriction (23) on the new earth-pressure coefficient is severe. A comparison of the steady uniform flow velocity $u_{s}$ with the maximum permissible velocity $u_{m}$ shows that

$$
u_{s} \leq u_{m} \Longleftrightarrow|\zeta| \leq \phi
$$

That is, the steady uniform velocity is less than the maximum permissible velocity if and only if the slope-inclination angle modulus is less than the material's internal angle of friction. If the inclination angle exceeds this amount and the slope is long enough, the earth-pressure coefficients at the nose of the avalanche become undefined and the problem becomes ill-posed.

It is clear that the Mohr-Coulomb constitutive relation cannot support the shear stresses imposed at the base of the avalanche in many physical situations. This is seen as evidence that a further rate-dependent contribution to the constitutive relation must be included to obtain a universally applicable theory.

\section{ACKNOWLEDGEMENTS}

This research was supported by the DFG project SFB 298 "Deformation und Versagen bei metallischen und granularen Strukturen".

\section{REFERENCES}

Hutter, K. and R. Greve. 1993. Two-dimensional similarity solutions for finite-mass granular avalanches with Coulomb- and viscous-type frictional resistance. 7. Glaciol., 39(132), 357-372.

Savage, S. B. and K. Hutter. 1989. The motion of a finite mass of granular material down a rough incline. F. Fluid Mech., 199, 177-21.5.

Savage, S. B. and K. Hutter. 1991. The dynamics of avalanches of granular materials from initiation to run-out. Part I. Analysis. Acta Mech., 86(1-4), 201-223.

Tai, Y. C. and J. M. N. T. Gray, 1998. Limiting stress states in granular avalanches. Ann. Glaciol., 26. (see paper in this volume).

Voellmy, A. 1955. Über die Zerstörungskraft von Lawinen. Schweiz. Bauztg., $73(12), 159-162$.

Whitham, G.B. 1974. Linear and non-linear waves. New York, etc., John Wiley and Sons. 